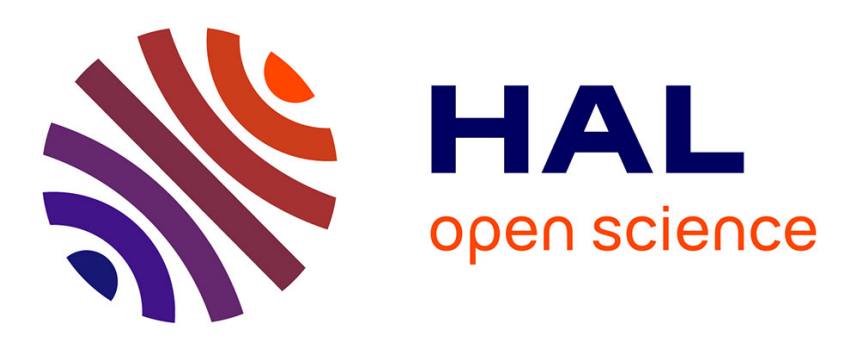

\title{
Prépositions et locutions prépositionnelles : la question du renouvellement grammatical
}

\author{
Benjamin Fagard
}

\section{To cite this version:}

Benjamin Fagard. Prépositions et locutions prépositionnelles : la question du renouvellement grammatical. Travaux de Linguistique: Revue Internationale de Linguistique Française, 2012, 64 (1), pp.161-189. 10.3917/tl.064.0161 . halshs-01241422

\section{HAL Id: halshs-01241422 \\ https://shs.hal.science/halshs-01241422}

Submitted on 10 Dec 2015

HAL is a multi-disciplinary open access archive for the deposit and dissemination of scientific research documents, whether they are published or not. The documents may come from teaching and research institutions in France or abroad, or from public or private research centers.
L'archive ouverte pluridisciplinaire HAL, est destinée au dépôt et à la diffusion de documents scientifiques de niveau recherche, publiés ou non, émanant des établissements d'enseignement et de recherche français ou étrangers, des laboratoires publics ou privés. 
Prépositions et locutions prépositionnelles : la question du renouvellement grammatical

\title{
Benjamin Fagard
}

\begin{abstract}
Résumé
Nous étudions ici l'équivalence ou la non-équivalence entre prépositions simples et complexes, à partir d'une comparaison sur corpus de la préposition dans et des locutions prépositionnelles au sein de, au milieu de, d'après, lors de, au cours de et au cœur de. Le principal corpus utilisé ici est constitué d'un mois du journal Le Monde, catégorisé et encodé dans le cadre de l’ANR «Spatial Framing Adverbials », et complété par d’autres corpus, écrits (diachroniques : base Frantext et DMF) et oral (Valibel). Afin de déterminer dans quelle mesure il peut y avoir une concurrence distributionnelle entre les morphèmes grammaticaux simples (dans) et complexes (les locutions prépositionnelles), nous les comparons du point de vue de la fréquence, du sens et de l'effet de la position syntaxique. Les résultats montrent une correspondance fonctionnelle très limitée.
\end{abstract}

\begin{abstract}
I study here the question of the semantic and functional equivalence between simple and complex adpositions, drawing on a corpus study of the French preposition dans 'in' as compared with a series of complex prepositions: au sein de, au cour de 'at the heart of', au milieu de 'in the middle of', d'après 'according to', lors de, au cours de 'during'. The main corpus I used is composed of one month of the French newspaper Le Monde, categorized and encoded by the ANR project "Spatial Framing Adverbials”, completing it with other written corpora (which include diachronic data: the Frantext and DMF databases) and an oral corpus (Valibel). In order to establish to what extent there might be a distributional competition between simple and complex grammatical morphemes, I compared them from the point of view of frequency, meaning and relationship between meaning and syntactic position. My results show a very limited functional equivalence between dans and the complex prepositions under study.
\end{abstract}

\section{Introduction}

Dans un article sur la préposition dans, Štichauer (2006) note qu'elle a remplacé en au 16 ème siècle dans des emplois du type «tant de royaumes réunis dans une vaste monarchie » (exemple de Gougenheim, 1970 : 54), et que cet emploi est désormais « atypique ». En effet, en français moderne, on dirait plutôt réunis au sein d'une vaste monarchie : qu'est-ce qui explique ce remplacement, tour à tour, d’une préposition simple (en) par une préposition composée (dans, formée sur de + intus) puis par une locution prépositionnelle (au sein de), dans un même contexte syntaxique ? La théorie de la grammaticalisation (depuis Meillet 1912 et Kuryłowicz 1965 jusqu’à, entre autres, Hopper \& Traugott 2003 ou encore Marchello-Nizia 
2006) permet semble-t-il de rendre compte assez bien de ce phénomène de renouvellement des catégories grammaticales, qui se fait d'une part par la grammaticalisation de morphèmes lexicaux ou grammaticaux, d'autre part par le figement de séquences complexes en locutions qui poursuivent parfois leur grammaticalisation et deviennent alors des éléments simples. C’est le schéma que propose Lehmann (1985) pour la catégorie des prépositions: les prépositions simples se grammaticalisent, et sont remplacées par des locutions prépositionnelles, qui elles-mêmes deviennent progressivement des prépositions simples, etc. La grammaticalisation même des prépositions simples, avec la désémantisation qu'elle entraîne, explique en effet le besoin que peuvent ressentir les locuteurs de leur substituer des constructions ayant un sémantisme plus précis (cf. Goyens, Lamiroy \& Melis 2002) : comme l’indique Haase (2001: 736), le degré de précision augmente avec la complexité morphologique ${ }^{1}$.

Il nous semble cependant que ce schéma n'est pas suffisamment clair. Se pose notamment la question de la coexistence entre morphèmes simples et complexes et du remplacement des unes par les autres : la disparition des morphèmes simples est-elle permise ou facilitée par l'existence des morphèmes complexes, ou bien l'apparition de morphèmes complexes est-elle au contraire déclenchée par la disparition des morphèmes simples ? Pour tenter d'apporter une réponse ou, plus modestement et de manière plus réaliste, une esquisse de réponse à cette question, nous proposons dans cet article une comparaison systématique des emplois d'une préposition simple (dans) et de quelques locutions prépositionnelles qui semblent être en concurrence (distributionnelle) avec cette préposition. Nous avons pour cela comparé dans à une série de locutions prépositionnelles dont les emplois recoupent en partie les siens : au sein de, au milieu de, d'après, lors de, au cours de et au cour de. Ces locutions ont été choisies sur la base du dictionnaire des synonymes du Crisco (http://www.crisco.unicaen.fr/). ${ }^{2}$ La comparaison a été faite à partir d'une étude sur corpus, à l'aide de la base utilisée dans le cadre de l’ANR-SFA (un mois du journal Le Monde), complétée par des excursus dans les bases DMF, Frantext et Valibel et par les ouvrages de référence : TLF-i, dictionnaire de l’Académie, Nicot, Godefroy, etc.

\footnotetext{
${ }^{1}$ L'auteur explique ainsi que la relation de localisation exprimée par dans est plus précise que celle qu'exprime à, mais moins que celles qui sont exprimées par des 'groupes prépositionnels' comme à l'intérieur de, dans le centre de, dans le cour de.

${ }^{2}$ Il va de soi que l'on aurait pu ajouter d'autres locutions qui, dans certains contextes, peuvent commuter avec dans, ainsi il court dans le bois chaque matin / il court à travers le bois chaque matin. Cependant, le but de cet article n'était pas d'étudier tous les équivalents de dans, et il nous semble que les locutions fournies par le Crisco (auxquelles nous avons ajouté la locution au cœur de) permettent déjà de faire un bon tour d'horizon des rapports entre préposition simple et locution prépositionnelle.
} 
Cette comparaison reposera sur trois points : a) fréquence, b) sémantisme, et c) distribution (nous entendons par là le lien entre position syntaxique et sémantisme). En conséquence, nous présentons dans un premier temps le rapport de fréquence existant entre dans et les locutions choisies (section 2). Dans un deuxième temps, nous décrivons les emplois de chaque locution, en montrant en particulier leur degré de polysémie et les domaines sémantiques dans lesquels elles peuvent être employées (espace, temps, etc.) (section 3). Dans un troisième temps, à partir des données ainsi rassemblées, nous mesurons le degré de proximité sémantique de chaque locution avec dans (section 4), et montrons en conclusion que la concurrence distributionnelle entre dans et ces locutions est en fait assez limitée.

\section{La fréquence}

L’importance de la fréquence pour l'ensemble des phénomènes linguistiques a été notée de longue date - avec par exemple les études de Zipf (1949) - et est désormais largement reconnue (cf. par exemple Ellis 2002, Diessel 2007). C'est particulièrement vrai pour les phénomènes de grammaticalisation, comme l’ont noté entre autres Bybee \& Hopper (2001), et Hopper \& Traugott (2003). De ce point de vue, si la grammaticalisation est un mouvement de figement qui touche non des mots isolés mais des constructions (cf. entre autres Traugott 2003, Himmelmann 2004, Gisborne \& Patten 2011, , Koch 2012), on pourra se demander quel intérêt il y a à étudier des constructions isolées de leur contexte : c'est la raison pour laquelle nous chercherons autant que possible à identifier les contextes d'emploi prototypiques des locutions à l'étude. ${ }^{3}$

Si l'on fait abstraction d'autres sources de fréquence (on pense par exemple aux noms propres), plus un élément s'est grammaticalisé, plus il est fréquent, et inversement. Nous partons donc du principe qu’il doit y avoir une corrélation entre la fréquence des prépositions et locutions prépositionnelles étudiées ici et leur degré de grammaticalisation, et que par ailleurs une fréquence semblable pour des éléments proches fonctionnellement indique un niveau de grammaticalisation similaire.

La fréquence d'emploi de dans dans le sous-corpus Le Monde à notre disposition est de 16355 occurrences sur 2406252 mots, soit une fréquence relative de 6800 occurrences par

\footnotetext{
${ }^{3}$ Štichauer (2006) note ainsi qu'une préposition « est le plus souvent employée [...] dans des constructions syntaxiques présentant une certaine régularité ». Son affirmation concerne les emplois locatifs, mais reste valable pour les autres. Un complément utile à notre enquête 'manuelle' sur corpus aurait été une étude statistique sur le modèle de Gréa (2005) ou encore de Blumenthal (2008).
} 
million environ. Cette fréquence assez élevée est attendue, et comparable à celle d’autres mots grammaticaux. ${ }^{4}$ La comparaison avec d'autres corpus de genres très différents (Frantext comprenant différents genres, Valibel de l'oral plus ou moins spontané) semble indiquer que cette fréquence est assez représentative de l'emploi de dans en français moderne (Tableau 1).

\begin{tabular}{|l|l|l|l|}
\hline & Tout Frantext 1950-2007 & Le Monde (un mois) & Valibel (sous-corpus) \\
\hline occurrence de dans & 444787 & 16355 & 3212 \\
\hline nombre de mots du corpus & 60219853 & 2406252 & 450722 \\
\hline $\begin{array}{l}\text { fréquence relative } \\
\text { [par million de mots) }\end{array}$ & $7386,05^{5}$ & 6796,88 & 7126,34 \\
\hline
\end{tabular}

Tableau 1 : Fréquence de la préposition dans dans différents corpus.

Si l'on compare cette fréquence avec celle des locutions prépositionnelles retenues, en revanche, la différence est importante. En effet, tandis que les différences entre prépositions simples sont relativement limitées (cf. note 1), celle que l'on peut observer entre dans et les locutions prépositionnelles partiellement synonymes est très importante : ces dernières sont entre 19 et 157 fois moins fréquentes (Tableau 2).

\begin{tabular}{|c|c|c|c|}
\hline locution & nombre d'occurrences prépositionnelles ${ }^{6}$ & \begin{tabular}{|l} 
fréquence relative \\
[par million de mots)
\end{tabular} & rapport / dans \\
\hline lors de & 854 & 354,91 & $1 / 19$ \\
\hline au cours de & 431 & 179,12 & $1 / 38$ \\
\hline au sein de & 313 & 130,08 & $1 / 52$ \\
\hline d'après & 111 & 46,13 & $1 / 147$ \\
\hline au cœur de & 111 & 46,13 & $1 / 147$ \\
\hline au milieu de & 104 & 43,22 & $1 / 157$ \\
\hline
\end{tabular}

Tableau 2 : Fréquence des locutions prépositionnelles étudiées ici, dans le (sous-)corpus Le Monde.

\footnotetext{
${ }^{4}$ Notamment les prépositions pour, par, sur et avec, dont la fréquence dans Frantext (tout de 1950 à 2007) sont d'environ 3000 à 5000 par million de mots ; les prépositions de et $a$, en revanche, ont des fréquences bien plus élevées, de l'ordre de 23000 pour à et 36000 pour de.

${ }^{5}$ La répartition chronologique des fréquences de dans dans ce sous-corpus est par ailleurs assez homogène (de 6900 à 8400 occ. par million de mots), mais la variation au sein de ce corpus reste plus grande que celle qu'on observe entre corpus - ce qui est un élément de plus pour dire que la fréquence observée dans le corpus $L e$ Monde est représentative.

${ }^{6}$ Les chiffres indiqués dans cette colonnes sont à distinguer du nombre d'occurrences de chacune des séquences graphiques, qui est légèrement suppérieur (lors de : 869, au cours de : 446, au sein de : 323, d'après : 127, au cœur de : 127, au milieu de : 109).
} 
Comme on l'a dit plus haut, étant donné l'importance de la fréquence pour de nombreux aspects du langage (Bybee \& Hopper 2001 : 2-3), on s’attend bien à trouver des différences importantes dans l'emploi de dans et des locutions étudiées. Dans la section suivante, nous proposons une brève description sémantique de ces éléments.

\section{Sémantisme comparé de dans et des locutions prépositionnelles retenues}

Pour les locutions, nous proposons ci-dessous une brève description sémantique. Pour dans, nous partirons ici de la description donnée dans Fagard \& Sarda (2009).

\subsection{La préposition dans}

La préposition dans, dont on trouve quelques occurrences (sous la forme dens, denz) en ancien français mais qui ne s'impose réellement qu'au $16^{\text {ème }}$ siècle, est en français moderne très polysémique, avec différents emplois spatiaux (dès les premières occurrences, cf. (1-2), et encore aujourd'hui), y compris métaphoriques (3), mais aussi temporels (4), ainsi que toute une série d'emplois abstraits (5a-c) :

$[1]^{7}$ il vint en très grande compaignie et mist bien dans la ville deux mille hommes d'armes (Commynes, Mémoires, p. 56, fin XVe) (base DMF, tiré de Fagard \& Sarda 2009)

[2] Les autres [soutiennent] que nous sommes nourris naturellement dans le ventre de noz meres, ainsi que les plantes, comme Crattipus (Pierre Boaistuau, Bref discours de l'excellence et dignité de l'homme, 1558, p. 40). (base Frantext, tiré de Fagard \& Sarda 2009)

[3] La répression en Syrie place la Turquie dans une position délicate (Le Monde, 10 juin 2011)

[4] L'alerte sur les concombres, les salades et les tomates doit être levée dans la journée. (Le Monde, 10 juin 2011)

[5] a. Dans l'affaire Tapie, la ministre de l'économie risque une enquête pour abus d'autorité, susceptible de compromettre sa candidature au FMI. (Le Monde, 10 juin 2011)

b. Google se renforce à nouveau dans la publicité en ligne (Le Monde, 10 juin 2011)

c. A Paris, Nicolas Hulot marque des points dans la primaire écologiste (Le Monde, 10 juin 2011)

Cette diversité sémantique est à mettre en lien avec sa fréquence élevée, notée plus haut.

3.2. Les locutions formées sur adverbe ou préposition

\subsubsection{La locution d'après}

\footnotetext{
${ }^{7}$ La source des exemples est indiquée, sauf lorsqu'il s’agit de notre corpus de base (un mois du Monde). Par ailleurs, pour les exemples en français moderne avec une locution prépositionnelle, nous notons systématiquement si la glose par «dans » nous semble possible : cela concerne, comme on le verra, une minorité d'exemples.
} 
La préposition après apparaît au $10^{\text {ème }}$ siècle. La locution d'après, elle, n’apparaît que vers 1670 d'après le TLF-i, et d'après + $N$ (ou GN) a initialement le sens de « suivant la norme, le modèle de ", également attesté pour après $N$ (ou $G N$ ) au $16^{\text {ème }}$ siècle. On peut préciser quelque peu : la séquence d'après (ou d'apres) est plus ancienne, puisqu'elle apparaît dès le début du $16^{\text {ème }}$ siècle, mais ne doit pas être interprétée comme une locution avant le milieu du $17^{\text {ème }}$, la première attestation que nous avons repérée dans la base Frantext datant de 1663 (6).

[6] Mais lorsque vous peignez les hommes, il faut peindre d’après nature. (Molière, La Critique de l'École des femmes, 1663, p. 351-353)

Les premières occurrences (dans la base Frantext) sont toutes de Molière, et formées sur le même modèle: peindre d'après nature ou encore, avec une variation minimale, d'après lui (i.e. le modèle), comme en (7) ; chez Madame de Sévigné, on trouve d'après nature mais aussi d'après le naturel (8). On peut se demander si d'après chez Corneille est utilisé comme locution ou comme construction libre (9).

[7] Car vouloir contrefaire un comédien dans un rôle comique, ce n’est pas le peindre lui-même, c'est peindre d'après lui les personnages qu'il représente, et se servir des mêmes traits et des mêmes couleurs qu'il est obligé d'employer aux différents tableaux des caractères ridicules qu’il imite d’après nature (...) (Molière, L’Impromptu de Versailles, 1673, p. 393-396)

[8] « ma foi, ma mère, vous voilà bien. » Vous entendez ces tons-là ; et pour les paroles, elles sont d'après le naturel. (Mme de Sévigné, Correspondance, 1675, p. 230, t. 1)

[9] Et si Léon devoit l'empire à votre appui, Lui qui vous y feroit le premier d'après lui, Auriezvous à rougir de l'en avoir fait maître, Seigneur, vous qui voyez que vous ne pouvez l'être ? (Pierre Corneille, Pulchérie, 1682, p. 434-435)

En français moderne, d'après le TLF-i, la locution d'après exprime «la norme, le modèle auquel on se conforme », ou encore "l'indice ». Sur la base du corpus Le Monde, on peut poser deux emplois principaux, où d'après introduit un 'point de référence', ou encore la 'source d'une information, d'une connaissance.'

Dans le premier cas, le point de référence peut constituer une norme acceptée par la communauté (10), une source matérielle (11-13), ou encore une source d’inspiration, de transposition, ou un modèle artistique (14) :

[10] D’après la loi, cependant, le gouvernement a les pleins pouvoirs jusqu'à ce qu'un nouveau premier ministre soit élu par le peuple.

[11] Cette nouvelle édition, dirigée par Jean-Pierre Sicre, et très finement illustrée d'après les maquettes originales d'Alain Meylan, vient à point (...). 
[12] On pourra compléter ce panorama national par le petit livret de Noël Simsolo : archives sonores de La Nouvelle Vague, deux disques compacts faisant entendre les voix de Truffaut, Godard, Resnais, Rohmer, d'après dix émissions de France Culture en 1987.

[13] Sa correspondance avec Maupassant est aujourd'hui scrupuleusement rééditée par Martine Reid d'après les autographes $(\ldots)$.

[14] Fabrice Luchini dit « L’Arrivée à New York » d'après Voyage au bout de la nuit, de Louis Ferdinand Céline.

Dans le second cas, d'après introduit la source de la connaissance (15), avec de nombreuses variantes : la source peut être une personne comme ci-dessus, mais aussi un groupe («les enquêteurs »), un institut («Eurostat»), un ensemble indéterminé (« des rumeurs de plateau »), un document ou un article (« un sondage ») etc., et il peut y avoir discours rapporté direct («briller une flamme... »), indirect ou indirect libre.

[15] D’après le ministre israélien des affaires étrangères, Shlomo Ben Ami, M. Clinton a donné, mercredi, aux négociateurs « les paramètres d'un accord » (...).

[16] D’après les enquêteurs, la victime aurait tenté de contrôler l'identité de deux personnes qui s’apprêtaient à partir en voiture, et qui lui ont tiré dessus presque à bout portant.

[17] D'après Eurostat, le taux de chômage s'élevait, en septembre, à 8,3\% en Allemagne et 6,2\% dans l'ouest du pays, contre 9,5\% en France.

[18] D’après des rumeurs de plateau, il paraît que le T-rex de 9 tonnes et le Spinosaurus de 12 tonnes vont se livrer un combat de géants dans JP3.

[19] D’après un sondage publié jeudi par le quotidien Yédiot Aharonot, M. Nétanyahou apparaît comme le meilleur candidat à droite (...). (glosable par « dans »)

[20] Bouffi, un bras dans le plâtre, la mémoire esquintée, il fait pourtant, d'après Ehrlich, «briller une flamme au fond de ses yeux », et obtient un triomphe qui sera un adieu.

En tout état de cause, le complément de la locution est ce qui permet au locuteur d'affirmer ce qu'il affirme :

[21] Aujourd'hui, d'après les sondages, M. Gore a convaincu plus de la moitié de l'opinion que les voix méritaient d'être recomptées. ${ }^{8}$

[22] D’après les chiffres des RG, près de la moitié des décès enregistrés de 1989 à 1998 résultaient de bagarres entre jeunes, pour des motifs dérisoires.

\footnotetext{
${ }^{8}$ Si la glose par dans apparaît ici moins acceptable, c'est sans doute, comme le suggère un relecteur anonyme, que le sondage est ici vu sous sa 'facette informationnelle' uniquement, tandis qu'il est vu aussi comme une entité matérielle (un texte publié dans un journal) en (19). L’emploi de dans serait dans ce cas permis par la matérialité du régime.
} 
On assiste donc à un mouvement de subjectification : de fabriquer, peindre qqch d'après qqch d'existant à d'après $X$, (je peux dire que/on peut dire que) $Y$. Ces emplois subjectifs où d'après pourrait être glosé par «selon » sont très loin des emplois de dans ; ils sont les emplois les plus fréquents de la locution d'après, formant environ les deux tiers des occurrences. Un élément de confirmation de ce mouvement de subjectification est la proportion élevée de compléments humains. Enfin, un cas particulier de cet emploi est la construction du type ([on peut dire que] X est Y d'après Z, Z étant une mesure de X) :

[23] L’état de trois des cinq malades s’est amélioré, selon des critères neurologiques et psychiatriques et aussi d’après des examens objectifs du métabolisme des cellules de la région.

[24] « L’ordinateur est la paillasse de l'étudiant en sciences humaines », insiste Michel Kaplan, président de Paris-I. D’après ses calculs, le coût annuel d'un élève d'école primaire se monte à 26000 francs, tandis qu’un étudiant de son université dispose de 22000 francs.

Un dernier emploi, marginal dans notre corpus, est celui où d'après introduit l'explication d'un nom (X est appelé Y d'après Z) (25) :

[25] Plus classiques, le récit de l'établissement de la puissance celte, l'éclosion d'une civilisation propre appelée laténienne, d'après le nom du lieu-dit (La Tène) de la commune de Marin-Epargnier dans le canton de Neuchâtel, en Suisse (...).

Ces emplois se répartissent comme suit : l’introduction de la prédication seconde est la plus fréquente, avec près des deux tiers des emplois, tandis que les emplois où d'après introduit un point de référence forment environ un tiers des occurrences, et que le dernier type est statistiquement négligeable.

\subsubsection{La locution lors de}

La locution lors de est formée sur l'adverbe lur "alors, à ce moment », qui apparaît en français au début du $12^{\text {ème }}$ siècle sur la base de latin vulgaire illa hora «à cette heure » > lore, avec chute du $e$ final puis suffixation d'un -s adverbial. La locution elle-même apparaît d'après le TLF-i à la fin du $16^{\text {ème }}$ siècle. ${ }^{9}$ Ses emplois en français classique et jusqu'en français moderne sont toujours temporels; c’est également le cas dans notre corpus, où la locution lors de ne présente que des emplois temporels, avec pour régime un GN temporel

\footnotetext{
${ }^{9}$ Une recherche dans la base DMF nous a permis de confirmer que toutes les séquences lors + de en moyen français ne sont pas à analyser comme une locution.
} 
(26-27) ou bien un GN désignant un procès ou un événement ${ }^{10}$ : réunion, conférence de presse (28-29).

[26] Le déficit des comptes publics italiens s'est établi à environ 35,74 milliards d'euros sur les onze premiers mois de l'année 2000, en hausse de 22,5\% sur le déficit affiché lors de la même période de 1999, a annoncé vendredi le ministère du Trésor. (glosable par « dans »)

[27] Vendredi, lors des dernières minutes de la séance, la progression de l'indice CAC 40, qui oscillait autour de $0,50 \%$ depuis la mi journée, a fondu pour se réduire en clôture à un misérable gain de $0,01 \%$ ! (glosable par «dans »)

[28] La coupe a commencé à déborder en mai, lors d'une petite réunion du Syndicat des avocats de France (SAF, gauche), qui a contaminé une assemblée générale du barreau avant les vacances.

[29] Cette opération a, comme fin 1999, été organisée pour limiter les risques d'accidents de la route durant la nuit du réveillon, a précisé $M$. Gayssot lors d’une conférence de presse sur la sécurité routière. (glosable par «dans »)

La locution opère alors un repérage temporel d’inclusion dans la période désignée par le GN temporel ou correspondant à l'intervalle temporel pendant lequel se déroule le procès ou l'événement. Même avec un régime désignant prototypiquement (ou potentiellement) un intervalle de temps, c’est plutôt la lecture 'événement’ qui est déclenchée par la locution, comme pour soirée dans les exemples suivants :

[30] L’affrontement entre deux bandes rivales avait commencé lors d'une soirée pour le Téléthon (...)

[31] Une rencontre « coup de foudre » pour lui, devenue « une très jolie histoire » pour elle, lors d'une soirée organisée par l'ami Eric Dahan, auteur à Libération des chroniques hebdomadaires du Paris nocturne dont Vincent McDoom est un héros récurrent.

Par ailleurs, il ne s’agit pas nécessairement d'un événement (ou d'un procès) unique (exemples 32-33).

[32] Onze lance-roquettes, provenant de l'ex-Yougoslavie et susceptibles d'être utilisés lors d'attaques de fourgons de transport de fonds, ont été découverts, mardi 28 novembre, dans un garage automobile à Nanterre (Hauts-de-Seine). (glosable par «dans », avec ajout d'un article indéfini)

[33] En Asie du Sud-Est, quatorze personnes ont été tuées lors d'attentats à la bombe contre la cathédrale de Djakarta et plusieurs églises indonésiennes. (idem)

Du point de vue diachronique, on peut remarquer une double évolution : d'un côté l'apparition (assez limitée) de compléments purement temporels (de type date ou durée, comme dans les exemples 26-27 ci-dessus), avec donc un sens de localisation temporelle apparemment renforcé, de l'autre un glissement du temporel au modal, avec des possibles

\footnotetext{
${ }^{10}$ La définition que nous adoptons pour événement et procès n'est pas lexicale : un même terme peut désigner selon le contexte un événement (c'est-à-dire, pour nous, une activité vue comme ponctuelle, quelque chose qui «a lieu ») ou un procès (c'est-à-dire une activité vue comme procédant, au sens étymologique, ou encore comme ayant des divisions temporelles internes et une progression) ; voir Huyghe (2012) pour plus de détails.
} 
lectures causales de certaines occurrences, de la même façon que pour au cours de (voir cidessous).

\subsection{Les locutions de type $a u N d e$}

\subsubsection{La locution au cours de}

La locution au cours de est formée sur le substantif cours (du latin cursus « action de courir, voyage, course en mer ; cours des étoiles, d'un fleuve ; cours de la vie de quelqu'un ; poét., d'un sentiment »), qui d'après le TLF-i apparaît en français au début du $12^{\text {ème }}$ siècle avec le sens d' « action de courir, déplacement » puis de «trajet d'un bateau », de «mouvement des astres » et enfin de «déroulement (du temps), succession ». Le sens figuré de cours désignant un sentiment ou un état affectif n’apparaît que vers le milieu du $15^{\text {ème }}$ siècle. Le TLF-i indique que la locution prépositionnelle au cours de commence à se lexicaliser au $16^{\text {ème }}$ siècle, mais une étude dans la base du DMF montre que la séquence au cours de est apparue bien plus tôt, dès le $14^{\text {ème }}$ siècle, avec un emploi encore référentiel du substantif cours.

La locution au cours de est figée en français moderne, comme en atteste par exemple l'impossibilité de l'insertion : *au plein cours de, *au cours exact/précis de sont impossibles dans les exemples (34-35) ci-dessous. Les emplois notés dans le TLF-i pour les locutions formées sur cours (dans le cours de ; au cours de, en cours de) sont uniquement temporels : ces locutions y sont glosées par «pendant la durée de », avec des exemples du $19^{\text {ème }}$ et du $20^{\text {ème }}$ siècles.

Dans le corpus Le Monde, on observe effectivement assez peu de variation sémantique, la locution ayant surtout des emplois temporels, avec principalement deux types de compléments : a) dans un peu plus de la moitié des occurrences (222 sur 431), le complément est de type 'date’ ou 'durée’ : jour, mois, année... La locution semble alors indiquer simplement un rapport de concomitance ou d'intervalle, avec un sens donc apparemment purement temporel (34-35). L'emploi de au cours de avec un complément de ce type se trouve dès le français classique (36), avec une nuance de sens légèrement différente cependant.

[34] Nous, les Serbes, avons vécu la tragédie d’avoir traversé tant de guerres au cours de ce siècle.

[35] Interdit de voyage, il avait récupéré son passeport en mai après en avoir été privé à plusieurs reprises au cours de ces cinq dernières années. (glosable par « dans »)

[36] On dira quelque jour, ici jadis fut Troye, Là son grand Ilion enclos de plus de tours, Qu'au cours de l'an entier on ne conte de jours. (Frantext, Antoine De Montchrestien, Hector, 1604, p. 64-65) 
Dans le reste des occurrences (209 sur 431), la locution a un régime de type 'procès', qui désigne aussi, généralement, un intervalle temporel, par métonymie de la durée requise pour l'exécution du procès. Le sens de la locution est alors temporel, mais avec souvent une inférence modale causale ou concessive, et on peut gloser au cours de $Y$ par 'à l'occasion de $\mathrm{Y}^{\prime}$, 'en raison de $\mathrm{Y}^{\prime}$ ou encore 'malgré $\mathrm{Y}$ ', 'bien que Y ait eu lieu' (37-40) :

[37] TROIS POLICIERS ont été blessés, jeudi matin 7 décembre, au cours d'affrontements entre les forces de l'ordre et des groupes de militants antimondialisation aux abords du centre des expositions Acropolis, à Nice. (glosable par «dans », avec ajout d'un article indéfini)

[38] Dira-t-on que l'armée française n’a pas torturé ou / et abattu que des combattants de l'armée de libération, à Alger, mais aussi des civils, hommes, femmes, enfants, au cours d'expéditions punitives, et ce, sur tout le territoire algérien ? (glosable par « dans », avec ajout d'un article indéfini)

[39] A partir d’une histoire réelle, parfois développée au cours d'improvisations, il écrit et dirige.

[40] Plus tard, il a été décoré pour la mise hors de combat, " au cours d’un accrochage ", de ce fellagha de haut rang.

On notera enfin que, lorsque le régime de la locution est une période, elle peut ne pas être conceptualisée comme formant un tout homogène :

[41] La fréquence d’El Niño s’est accélérée au cours du XXe siècle.

On peut résumer ces deux emplois différents comme suit :

a) $\mathrm{X}$ au cours de $\mathrm{Y}$ indique que l'événement $\mathrm{X}$ s'est produit au cours de l'intervalle temporel Y

b) $\mathrm{X}$ au cours de $Y$ indique que l'événement $\mathrm{X}$ s'est produit au cours du procès $\mathrm{Y}$, et probablement en raison de ce procès ou du moins en lien avec ce procès (lecture modale).

Il faut noter que l'emploi (b) n’est pas réellement lexicalisé : il s'agit d'une interprétation pragmatique en discours.

\subsubsection{La locution au milieu de}

La locution au milieu de est formée sur le substantif milieu (sur lieu, du latin locus), qui apparaît dès l'ancien français. Au début du $12^{\text {ème }}$ siècle apparaît la séquence el millieu de « au sein d'un groupe de personnes ", " au cours de » (temporel), d'après le TLF-i ; la locution au milieu de apparaît au début du $14^{\text {ème }}$ avec le sens « à mi-distance des extrémités, au centre de » puis au $16^{\text {ème }}$ avec un sens temporel («à un moment d’une durée également éloigné du début et de la fin ») ou figuré (par exemple au meilleu du bruit et du tumulte des armées), y compris sous la forme au beau milieu de. 
L'emploi central noté par le TLF-i pour la locution au milieu de est le sens spatial « au centre de, dans la partie centrale de », ou encore « à l'intérieur de, à un endroit relativement éloigné des bords, de la périphérie de ». On trouve en outre un sens temporel, avec des régimes indiquant des périodes de temps diverses ou une métaphore spatiale (au milieu du chemin de la vie). Le TLF-i note également un certain nombre de sens ou d'emplois abstraits, avec différentes nuances sémantiques en fonction du type de complément, ces derniers allant de l' « ensemble de pensées » aux personnes, aux circonstances extérieures et à «l'état physique ou moral de quelqu'un ». La locution peut alors être glosée par « au sein (d’un groupe), en compagnie, en société de », « entouré de, accompagné de », « au cœur de ». Enfin, il y a également des emplois modaux, où la locution prend une valeur adversative, comme dans $a u$ milieu de tout cela «malgré tout cela ».

D'un point de vue morpho-syntaxique, la locution semble assez figée, mais le TLF-i note l'existence des formes au beau milieu de et tout au milieu de (ou encore tout au beau milieu de).

Dans notre corpus, les emplois de au milieu de sont principalement de trois types : temporels, spatiaux et 'inclusifs'. Les emplois temporels forment près de la moitié des occurrences, que le complément désigne un intervalle temporel (42-43), une représentation artistique (44) ou un autre procès (45) :

[42] La présentation de Geri, une ex-Spice Girl, de Molly Dineen (1999), film consacré à l'une des chanteuses du groupe féminin britannique - qu'elle a « osé » quitter au milieu de 1998 -, pouvait faire craindre le même travers.

[43] Invité à écrire une pièce à l'usage d'une compagnie jouant devant des adolescents, dans les lycées et écoles, Bond rappelle que « comme tous les gens en vie au milieu de ce siècle ou nés depuis, je suis un citoyen d'Auschwitz ».

[44] Au milieu d'Incassable, Elijah Price (...) saisit péniblement (...) une bande dessinée (...). (grammatical mais pragmatiquement incorrect si glosé par dans)

[45] Nous continuons de penser que le risque de récession est faible, mais l'économie américaine est au milieu de ce que l'on pourrait appeler un atterrissage mouvementé, avec une croissance inférieure à $3 \%$.

Les emplois spatiaux, qui forment également près de la moitié des occurrences, ne désignent généralement pas une configuration géométrique, conformément à ce que note Gréa (2005 et 
sous presse ${ }^{11}$ ), et semblent parfois relever de mécanismes plutôt fonctionnels que proprement spatiaux, avec des nuances contrastives plus ou moins marquées, i.e. avec une opposition plus ou moins nette entre le sujet du verbe et le régime de la locution (cf. elle et les chevaux en 46, je/Noir et Blancs en 47, Julia et cuvettes sales en 48, Marcos et les journalistes en 49) :

[46] Elle a grandi au milieu des chevaux que son père dressait.

[47] Dans le hall de l'université, j’étais la plupart du temps le seul Noir au milieu des Blancs.

[48] Tandis que Julia sanglote sans fin au milieu de cuvettes sales, un vieux poste TV crachouille les images d'un groupe de musiciens mariachis (particulièrement ringards) qui commentent l'action façon chœur antique : « Nicolas, le beau Nicolas, s’est conduit comme un ingrat ».

[49] Très à l'aise au milieu des journalistes qu'il avait convoqués deux jours avant, Marcos, la pipe à la bouche et le fusil en bandoulière, n’a pas raté son retour (...).

Cette nuance contrastive peut d'ailleurs se trouver avec des emplois non spatiaux (50-51) :

[50] Ceux d'entre eux qui avaient combattu sous l'uniforme français, et auxquels s’étaient joints des fonctionnaires et des civils, arrivèrent sur le territoire métropolitain au milieu des pires périls pour y connaître indifférence, mépris et mauvais accueil.

[51] Au milieu du concert d'appels à une coalition républicains démocrates au centre, plusieurs porteparole de la droite républicaine, comme Gary Bauer, ont tenu à rappeler que c’étaient leurs troupes qui avaient formé les bataillons électoraux de George W. Bush.

Il y a également quelques emplois que nous appelons 'inclusifs', et qui se rapprochent des emplois ‘ensemblistes’ de Hilgert (sous presse) :

[52] Au milieu des mastodontes que sont les titres régionaux Sud-Ouest en Aquitaine, Diario Vasco ou Correo en Espagne, Egunkaria, dont le siège est près de saint sébastien, est diffusé à 14300 exemplaires chaque jour (...).

Le sens de la locution, dans ce cas, n’est clairement plus spatial (ou alors de manière purement métaphorique).

\section{3..3.3. La locution au sein de}

Au sein de est une locution prépositionnelle formée sur sein, qui d’après le TLF-i apparaît en français au début du $12^{\text {ème }}$ avec le sens de « partie du vêtement qui recouvre la poitrine » puis "partie antérieure du thorax humain », y compris au figuré avec le sens de «siège des sentiments », et "partie inférieure, intime ». La locution apparaît, toujours d'après le TLF-i, dans la deuxième moitié du $16^{\text {ème }}$ siècle (1558), mais ni Nicot (1606), ni le Dictionnaire de l'Académie française (éditions 1694 et 1762) ne notent son existence. Il faut attendre le

\footnotetext{
${ }^{11}$ Il note en particulier que le choix entre au centre de et au milieu de n’est pas influencé par «la forme géométrique ou physique du complément ni sa dimension » mais par « sa plus ou moins bonne délimitation ».
} 
Dictionnaire critique de Féraud (1787-1788), qui donne entre autres l'exemple suivant, de Boileau :

[53] Mais une Église seule à ses yeux immobile,

Garde au sein du tumulte une assiète tranquile. (Boileau, Le Lutrin, 1674-1683)

Du point de vue du sens, le TLF-i donne pour au sein de les deux définitions suivantes :

a) Lorsque le complément est une chose physique, « au plus profond de, au milieu de »

b) Lorsque le complément est abstrait, « dans, parmi ».

Dans notre corpus, on trouve principalement des emplois abstraits, où au sein de indique le 'groupe social', le cadre ou le milieu dans lequel un événement se produit (300 occ., exemples 54-55).

[54] Elle a créé une tempête, un vent de panique au sein de la hiérarchie militaire, et c'est à ce momentlà que le sort de Bouteflika a fini d'être scellé. (glosable par « dans »)

[55] Sans ces collaborateurs de la justice, nous ne pouvons rien savoir des affaires qui se passent actuellement et des modifications de structure en cours au sein de la Mafia et au sein même des " familles ». (glosable par «dans »)

Il s'agit de loin de l'emploi le plus fréquent dans notre corpus, avec environ $95 \%$ des occurrences.

Les occurrences de au sein de avec un régime de type (plus ou moins clairement) 'spatial' sont rares : 13 en tout dans notre corpus. De plus, même quand le régime est spatial ou du moins constitue une entité ayant une étendue dans l'espace, la locution au sein de n'opère pas un repérage spatial, comme dans les exemples (56-61) ci-dessous : le pays, la planète, le club, les internats, la fondation désignent ici des communautés plutôt que des étendues spatiales.

[56] Catholiques et chrétiens orthodoxes, musulmans et juifs se côtoyaient au sein d'un pays où la Serbie, dont le rôle fédérateur perdurait depuis le Moyen Age, tentait de faire vivre ensemble Croates et Macédoniens, Slovènes, Monténégrins et Albanais, le Nord développé, sous l’influence germanique, et le Sud misérable, longtemps tributaire du Sultan. (glosable par « dans »)

[57] On se rendra compte à sa lecture qu’à presque trente ans d'écart les problèmes de survie d'une région au sein d'une planète en proie à la mondialisation demeurent. (glosable par « dans », ainsi que par «sur»)

[58] Faruk Suren, qui doit faire face à une forte opposition au sein de son club depuis la transformation de ce dernier en société anonyme en 1999, a répondu à ces accusations (...). (glosable par « dans »)

[59] Il faut apprendre à le connaître, le rencontrer souvent au sein de son club... (glosable par « dans »)

[60] (...) Roger Peyrefitte, élève des jésuites à Toulouse, a provoqué le scandale en décrivant les relations homosexuelles au sein des internats de garçons dans Les Amitiés particulières (...) (glosable par «dans ») 
[61] Je ne sais pas si c'est une bonne idée, mais nous devons y penser, soit au sein de la fondation, soit dans l'aménagement global de l'île, qui pourrait recevoir un certain nombre de créations qui, comme le Jeff Koons, ont une dimension urbaine.

On trouve enfin quelques occurrences où le régime de la locution est un concept abstrait : thématique, pensée... il nous semble que, dans ce cas encore, au sein de indique le cadre (abstrait) dans lequel s’inscrit ou apparaît un concept (62-64) :

[62] Trois « images », au sens fort, sont ainsi suivies dans le temps et dans l'espace, jusqu'aujourd'hui : la présence du général de Gaulle, la rhétorique de la « justice » appliquée à l’Allemagne, et la lente apparition de l'extermination des juifs d'Europe au sein de la thématique de la déportation.

[63] Et c'est donc qu'on s'est établi dans une pensée conceptuelle, au sein de laquelle, je l'ai souligné d'emblée, on bute partout sur l'énigme, et avec angoisse.

[64] Louis Mexandeau n’a pas su créer de dynamique au sein de la gauche plurielle, ni dans l'opinion publique, qui attend pourtant un maire de gauche à Caen », assurent M. Duron et M. Geindre. (pour ces trois exemples, la glose par "dans» ne semble pas exclue, mais maladroite, au moins pour les exemples 62 et 64)

\subsubsection{La locution au cœur de}

La locution au cœur de est formée sur le substantif cœur, qui apparaît dès l'ancien français sous la forme cor, cuer. Les premières occurrences de la séquence $a u+c œ u r+d e$ qui pourraient être interprétées comme formant locution sont assez anciennes, remontant au tout début du $15^{\text {ème }}$ siècle, mais coexistent longtemps avec l'interprétation non figée de la même séquence (dernier exemple ci-dessous) :

[65] Et ce vous poroit grandement couster de vos honmes, et vous en avés bien a faire, avant que vous soiiés au cor de vostre voiage (...) (Base DMF, Jean Froissart, Chroniques, 1400, p. 694)

[66] Et de fait vint en France, l'an mil IIJcXXIX en la ville d'Amiens, et au cuer de la grant esglise fit l'ommage de la duchié de Guyenne, le quel hommage fut fait bien grandement et solemnellement et de la franche et pure voulenté du dit Edouard et par le conseil et deliberation de ceulx de son sang et de tous les troys estas d'Engleterre. (Base DMF, Jean Juvenal des Ursins, Tres crestien, tres hault, tres puissant roy, 1446, p. 112, T.2)

[67] Et en tel estat vindrent en l'eglise de Sainct Jehan, qui est une des principales eglises et paroisses de Gand, et à l'entrée de celle eglise trouverent l'evesque de Tournay revestu, avecques les chanoines, chappelains et choreaulx d'icelle eglise, qui recuillirent le duc et ses freres moult devotement, et, en chantant himnes et cantiques devotes, les conduisirent jusques au cueur de l'eglise, et dont les formes d'icelluy cueur furent parez de tableaux, armez et timbrez des armes et timbres des chevaliers, de leurs motz, de leurs noms et de leurs devises. (Base DMF, Olivier de la Marche, Mémoires, 1470, p. 88, t. 2, Livre 1) 
[68] - Je me contente, dist-il, de l'amour que je sens en moy et de l'espoir qu'il y a au coeur des dames, mais, si je le sçavois, comme je l'espere, j'aurois si extresme contentement, que je ne le sçaurois porter sans mourir. (Base Frantext, Marguerite De Navarre, L’Heptaméron, 1550, p. 938)

L'emploi de au cœur de comme locution est signalé par le DMF, qui ne note cependant que la locution «au / en coeur d'hiver».

En français moderne, au cœur de coexiste encore avec des emplois non prépositionnels de la séquence mais fonctionne le plus souvent comme une locution prépositionnelle (cf. le peu de bruit obtenu à l'aide de la requête au cœur de dans notre corpus du Monde). Le TLF-i note plusieurs sens pour cette locution : un sens métaphorique ou figuré par référence «plus ou moins nette à la position quasi-médiane du cœur dans la poitrine », un sens temporel, et un sens abstrait (Au cœur de la vie humaine... ; Entrer au cœur des choses, du débat).

Dans notre corpus, on trouve environ un tiers d'emplois spatiaux, où la locution situe $\mathrm{X}$ au centre d'une région Y :

[69] UNE FORÊT, îlot de végétation isolé au cœur de la toundra. (glosable par « dans »)

[70] Un gros pâté d’habitations est aussi habité par des juifs au cour du quartier musulman. (glosable par «dans »)

Quelques occurrences, en outre, où il s’agit d’un espace métaphorique :

[71] [La Caisse des dépôts et consignations...] paraissait plonger au cœur de réseaux d’affaires disposant d'appuis et d'entrées au sein du pouvoir socialiste de l'époque.

[72] LONGTEMPS, les dossiers des anciennes colonies françaises étaient logés au cour de l'Etat, à l’Elysée même, où un confident du président cultivait ce jardin secret.

[73] Autre grand thème : le Salon nous invite à un « voyage au cœur des identités » autour de la question du masculin et du féminin en littérature de jeunesse.

Mais la plupart des occurrences - environ les deux tiers - sont des emplois purement abstraits, où la locution indique le cadre dans lequel un thème est à envisager. Ce cadre est le plus souvent un domaine lié à l'échange d’idées (discussion, débat, élection, roman) :

[74] La Charte européenne au cour de la contestation

[75] Dans chacun de ces cas, la télévision se trouve au cœur des décisions.

[76] Car c’est bien la responsabilité des femmes qui a été au cour des débats, la majorité reprochant à l'opposition de vouloir accorder une trop grande place aux experts (médecins...) dans la décision d'avorter, au risque de déposséder les femmes de leur liberté.

Cependant, il peut aussi s’agir de domaines ou processus divers : procédures d'évaluation, l'ancienne économie, trio fondateur...

Notre corpus ne contient en revanche que deux occurrences temporelles, alors que cet emploi semblait assez important en français préclassique : 
[77] ILS n’étaient pas très nombreux, il est vrai, ceux qui, au cour des années 50 pensaient que c’en était fini de l’Algérie française et que ce pays allait vers l’indépendance. (glosable par «dans »)

[78] Au cœur de la nuit polaire, en décembre, il souhaite partager l’intimité des campements de nomades. (glosable par «dans »)

\section{Sens et position}

Nous avons montré à l’occasion d'une étude des emplois de la préposition dans dans ce même corpus (Fagard \& Sarda 2009) que les emplois de dans dépendaient largement de sa position dans la phrase, et plus précisément du statut du syntagme qu'elle introduit: lorsque le syntagme prépositionnel est intégré, il a le plus souvent un sens spatial ou abstrait, mais lorsqu’il est extraposé, il a majoritairement un sens 'énonciatif', c’est-à-dire qu’il joue un rôle dans l'organisation du discours. Nous voudrions voir ici si cette caractéristique de dans se retrouve pour les locutions prépositionnelles sémantiquement proches, et mettons donc le sens de chacun de ces éléments en rapport avec sa position dans la phrase. Nous montrons ainsi que la correspondance fonctionnelle entre dans et chacune des locutions prépositionnelles étudiées n’est que très parcellaire.

Si l'on s'attache d'abord à une comparaison sémantique 'hors position', on voit que la ‘correspondance’ sémantique entre dans et les locutions choisies est en fait très limitée. Cela est vrai dans les détails des constructions attestées, comme on a pu le voir dans la section 3, mais aussi pour la répartition des emplois, comme on le voit dans le graphique 1 ci-dessous.

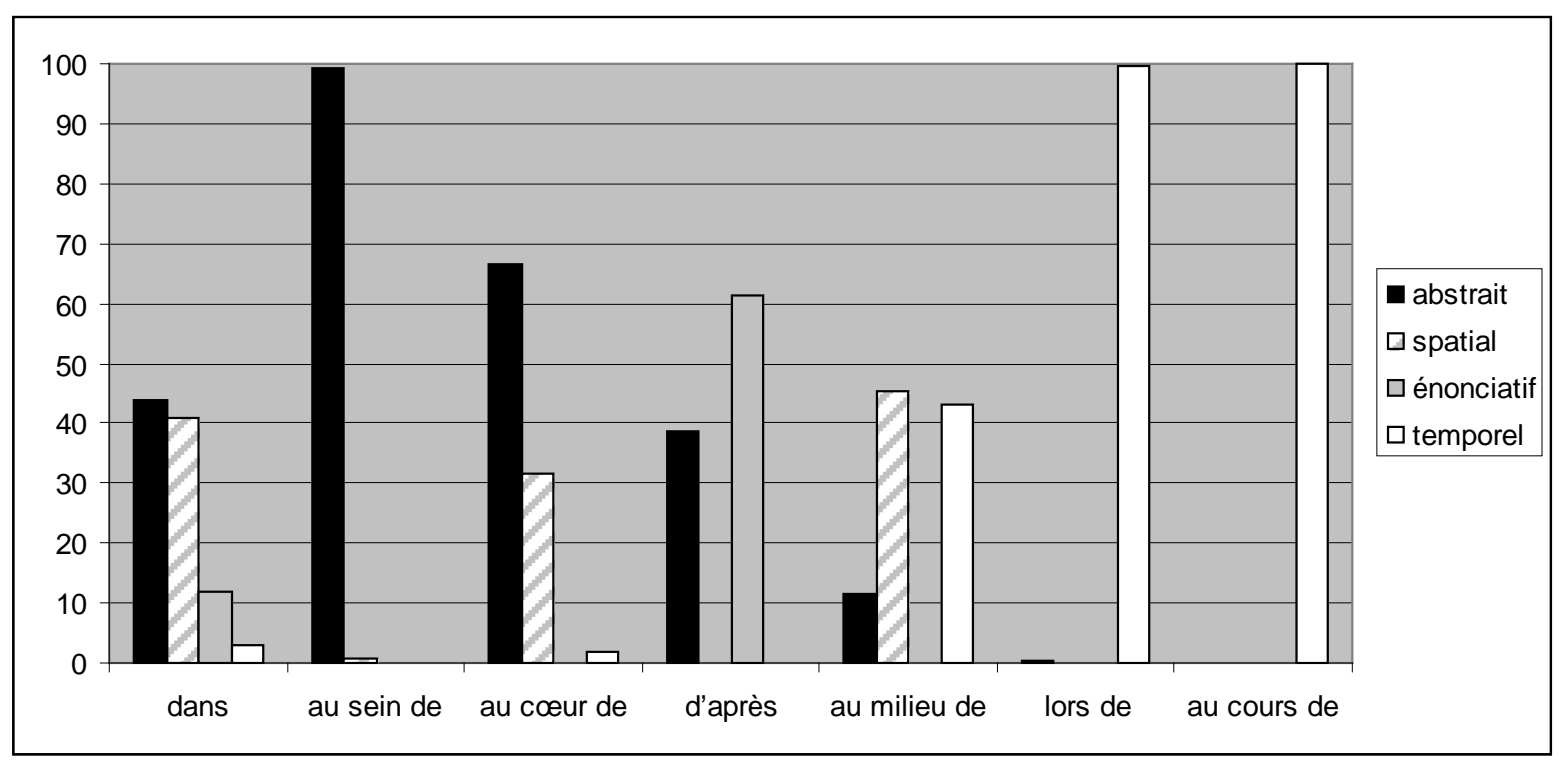

Graphique 1 : Répartition des emplois en domaines sémantiques dans le corpus Le Monde (en pourcentages). 
De plus, si l'on tient compte des fréquences réelles, l'absence de correspondance est plus évidente encore, comme on le voit dans le graphique 2 ci-dessous. Le seul domaine pour lequel on pourrait poser une équivalence de fréquence est en effet le domaine temporel, et ceci uniquement pour lors de et au cours de. S’il y a correspondance entre dans et les locutions prépositionnelles, ce n’est donc, dans la grande majorité des cas, que pour des constructions isolées.

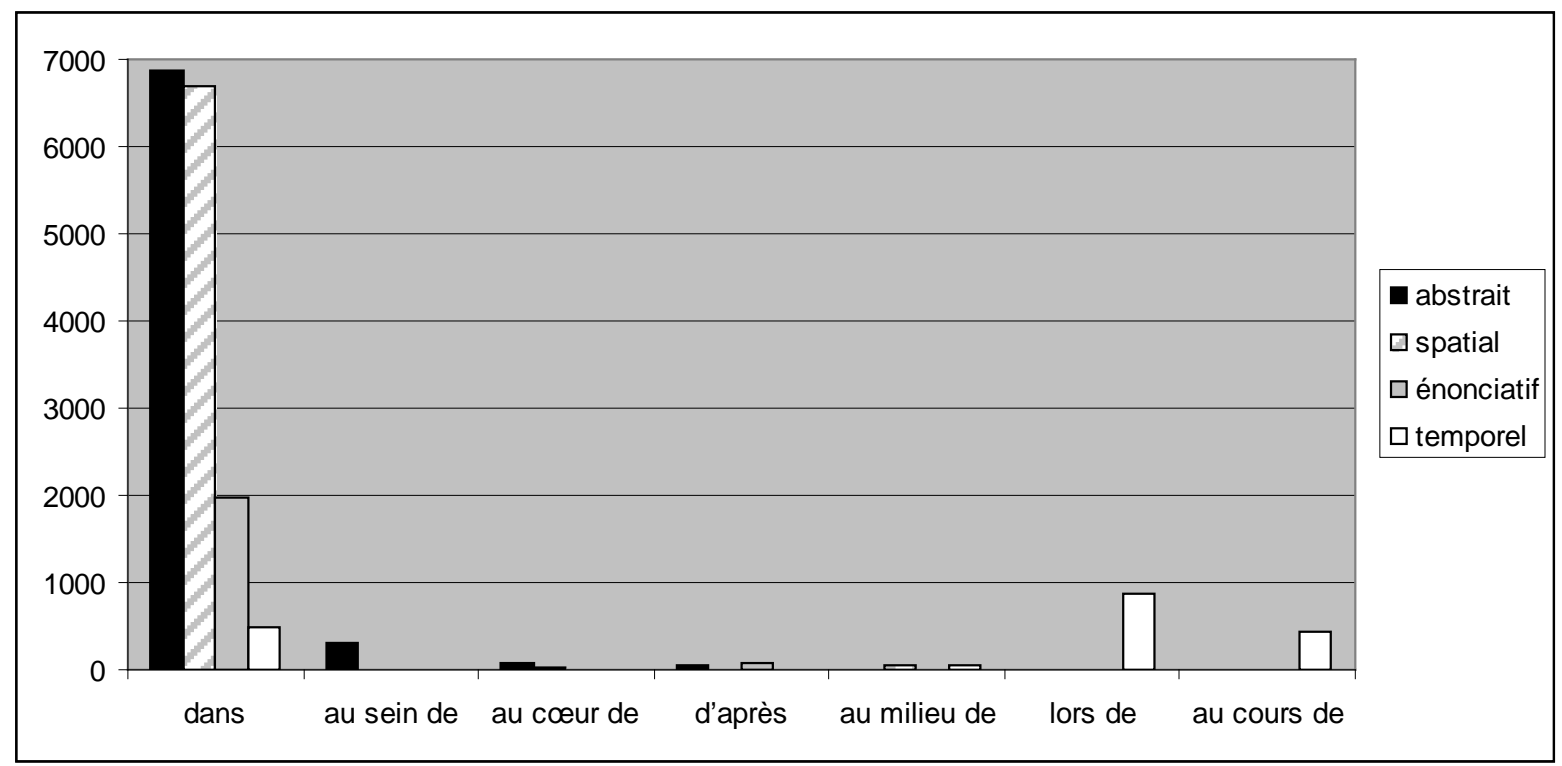

Graphique 2: Répartition des emplois en domaines sémantiques dans le corpus Le Monde (en nombre d'occurrences).

Du point de vue de la proportion des occurrences à l'initiale (c'est-à-dire en début de proposition), en revanche, la préposition dans a un fonctionnement comparable à la plupart des locutions prises en compte. Seules au sein de, qui est rarement à l'initiale, et d'après, qui est assez fréquemment à l'initiale, s'en distinguent nettement. Mais en fréquence brute, même d'après apparaît bien moins souvent à l'initiale que dans, comme on le voit dans le tableau 3 ci-dessous.

\begin{tabular}{|l|l|l|}
\hline & Nombre d'occurrences initiales & $\%$ initial \\
\hline dans & 1685 & 10,30 \\
\hline lors de & 88 & 10,21 \\
\hline au cours de & 61 & 14,09 \\
\hline d'après & 49 & 44,95 \\
\hline au sein de & 13 & 4,13 \\
au cour de & 9 & 8,11 \\
\hline au milieu de & 8 & 7,69 \\
\hline
\end{tabular}


Tableau 3 : Emploi de dans et des locutions prépositionnelles étudiées en début de proposition (fréquence brute et pourcentage des emplois), dans le corpus Le Monde.

D’un point de vue qualitatif, en revanche, on peut remarquer certaines similarités entre les emplois initiaux de dans et ceux des locutions prépositionnelles. D’une part, les emplois spatiaux sont souvent moins fréquents à l'initiale : ainsi, pour au milieu de et au sein de, les emplois spatiaux disparaissent totalement dans cette position. Malheureusement, il n'est pas possible de vérifier la significativité à l'aide du chi², car les effectifs sont trop faibles. Pour les autres locutions (du moins celles ayant des emplois spatiaux), on retrouve le même phénomène, mais de manière moins marquée. D’autre part, la seule locution réellement fréquente à l'initiale est d'après, dont les emplois sont les plus subjectifs et tendent le plus vers l'énonciatif. Ainsi, dans les exemples où le syntagme introduit par d'après se trouve en tête de proposition (comme en (22)), on peut considérer que ce syntagme introduit ce que Charolles (2005) appelle un cadre de discours : tout ce qui suit est à interpréter comme dépendant du point de vue énoncé par le syntagme prépositionnel, jusqu’à la fermeture du cadre ou l'ouverture d'un cadre successif. De ce point de vue, les syntagmes initiaux en d'après se rapprochent fonctionnellement de certains syntagmes initiaux en dans (cf. Fagard \& Sarda 2009 : exemples 10 et $11^{12}$ ). Cependant, ces deux types d'emplois sont bien distincts sémantiquement, puisque les syntagmes en d'après introduisent le point de vue et ceux en dans plutôt une modalité d'expression ou d'énonciation. ${ }^{13}$

\section{Conclusion}

Nous avons comparé les emplois d'une préposition simple et de locutions prépositionnelles 'synonymes', à partir d'un corpus écrit de français moderne. Le but de cette étude était de voir dans quelle mesure il y avait ou non 'concurrence' entre le morphème le plus grammaticalisé (dans) et les autres, afin d’avancer dans notre réflexion sur le renouvellement grammatical.

\footnotetext{
${ }^{12}$ C'est-à-dire les exemples suivants :

(10) Dans certains cas, les négociations ont permis aux syndicats d'obtenir des améliorations par rapport au texte initial, comme par exemple à Cléon. (corpus Le Monde) ;

(11) Dans un prologue un peu ampoulé, Renaud Ego convoque les ombres de Foucault, Deleuze, Merleau-Ponty ou Bachelard comme autant de béquilles virtuelles. (corpus Le Monde).

${ }^{13}$ Il faudrait, pour compléter cette étude, comparer une à une les fréquences à l'initiale pour chaque sens de toutes les prépositions simples et complexes prises en compte dans cet article. Les limites de place nous amènent malheureusement à garder cette intéressante piste de réflexion, suggérée par un relecteur anonyme, pour une prochaine étude.
} 
Nous avons montré qu'il n’y a pas de synonymie parfaite entre dans et les locutions analysées, mis à part dans quelques contextes très spécifiques : comme on l'a vu au cas par cas, sur les locutions dans les exemples 10 à 78 en français moderne, seule une vingtaine peuvent être glosées par « dans ». Par ailleurs, ces gloses impliquent parfois des modifications syntaxiques (dans introduisant nécessairement un groupe nominal précédé par un article) ou des problèmes d'interprétation (sémantico-pragmatiques); de plus, certaines locutions semblent plus éloignées sémantiquement, comme d'après et au milieu de (un seul exemple glosable par «dans »). On ne peut donc pas parler de remplacement de dans par ces locutions prépositionnelles. Certaines d'entre elles sont apparues en français à une date très ancienne, et pourtant elles ont un degré de grammaticalisation limité, comme on le voit à l'absence de réduction phonologique, à leur polysémie limitée et à leur fréquence encore faible. Certes, le remplacement d'une préposition par une autre n’est pas nécessairement rapide, ${ }^{14}$ ni complet : ainsi, si dans a remplacé en dans certains emplois, il n'en reste pas moins que en est encore employée en français moderne.

Tout cela semblerait indiquer que les locutions prépositionnelles remplissent, au moins en partie, un rôle parallèle à celui des prépositions simples : même si les prépositions simples sont parfois issues de locutions prépositionnelles, cela ne signifie pas que ces dernières soient ‘condamnées’ à se simplifier (c’est-à-dire à se grammaticaliser) pour devenir des prépositions simples. Par ailleurs, la simplification d’une locution prépositionnelle n'en fait pas nécessairement une concurrente directe d'autres prépositions simples (cf. la description du passage de du côté de à côté dans Fagard 2008). C’est là un trait bien connu de la grammaticalisation : il s’agit d'un phénomène linguistique qui ne va que rarement jusqu'à son terme.

Il faut tout de même signaler en conclusion que, parmi les locutions étudiées, une semble se distinguer et paraît avoir acquis des emplois assez proches de ceux de dans : il s'agit de d'après, qui est, comme nous l'avons vu dans la section 4, employée pour introduire des cadres de discours. Si elle ne remplace pas dans, c'est probablement que sa proximité avec dans est limitée à des contextes trop spécifiques ${ }^{15}$.

\section{Références}

\footnotetext{
${ }^{14}$ Cf. Štichauer (2006) : « une fois introduite dans la langue vers 1550, la préposition dans (dedans) s’impose relativement lentement. (...) Peu à peu, l'évolution est allée dans le sens d’une certaine ‘spécialisation’ des emplois (...)».

${ }^{15}$ Merci à un des relecteurs anonymes, qui m’a suggéré cette explication.
} 
Blumenthal P., 2008, « Combinatoire des prépositions : approche quantitative », Langue Française, 157, p. 37-51.

Bybee J. et Hopper P., 2001, Introduction, in BYBeE J. et HOPPER P., Frequency and the emergence of linguistic structure, Amsterdam / Philadelphia, John Benjamins, p. 1-24.

CharolLes M., 2005, « Framing Adverbials and Their Role in Discourse Cohesion: From Connection to Foward Labelling », in Aurnague M., Bras M., Le Draoulec A. et Vieu L. (eds.), SEM-05 Proceedings, Biarritz, p. 13-30.

Dictionnaire de l'Académie française, $1^{\text {ère }}$ édition, 1694, Paris, Coignard.

Dictionnaire de l'Académie française, $4^{\text {ème }}$ édition, 1762, Paris, Coignard.

DIESSEL H., 2007, « Frequency effects in language acquisition, language use, and diachronic change », New Ideas in Psychology, 25, p. 108-127.

ELLIS N., 2002, «Frequency Effects in Language Processing - A Review with Implications for Theories of Implicit and Explicit Language Acquisition », SSLA, 24, p. 143-188.

FAGARD B. et SARDA L., 2009, «Etude diachronique de la préposition dans », in FrANÇOIS J., Gilbert E., Guimier C. et Krause M. (eds.), Autour de la préposition, Caen, Presses universitaires de Caen, p. 225-235.

FAGARD B., 2008, « 'Côté' dégrammaticalisation - le cas des prépositions », in FAGARD B., PREVOST S., Combettes B. et Bertrand O. (eds.), Evolutions en français, Berne, Peter Lang, p. 87-104.

FERAUD J.-F., 1787-1788, Dictionnaire critique de la langue française, Marseille, Mossy.

Gisborne N. et PAtTen A., 2011, « Construction Grammar and grammaticalization », in NARROG H. et HeINE B. (eds), The Oxford Handbook of grammaticalization, Oxford : Oxford University Press, p. 92-104.

Gougenheim G., 1970, "Tant de royaumes réunis dans une vaste monarchie », in Deloffre F., Eskenazi A., PicARD J. et Regnier C. (eds.), Etudes de grammaire et de vocabulaire français, Paris, Picard.

Goyens M., LAMiRoy B. et MeLIS L., 2002, « Déplacement et repositionnement de la préposition à en français », Lingvisticae Investigationes 25, p. 275-310.

GrEA P., 2005, "Les locutions au centre / milieu de dans leur acception spatiale », Revue de Sémantique et de Pragmatique, 18, p. 87-112.

GREA P., (sous presse), «Le centre n’est pas au milieu (et inversement). Pour une approche phénoménologique et gestaltiste de la localisation », Corela. 
HaAse M., 2001, «Lokalkasus und Adpositionen », in Haspelmath, M., E. König, W. ÖSTERREICHER, et W. RAIBLE (eds), Language Typology and Language Universals, Vol. 1, Berlin / New York : Walter de Gruyter, p. 736-741.

HiLgert É., (sous presse), «Les prépositions ensemblistes et la question de leur emploi spatial », Corela.

HimMELMANN N., 2004, "Lexicalization and grammaticalization: Opposite or orthogonal? », in BISANG W. et al (eds), What makes grammaticalization, Berlin : Mouton de Gruyter, p. 21-42.

Hopper P. et TraugotT E., 2003 [1993], Grammaticalization, Cambridge, Cambridge University Press.

HUYGHE R., 2012, « Noms d’objets et noms d'événements : quelles frontières linguistiques ? », Scolia 26, p. 81-103.

Koch P., 2012, «Changement lexical et constructionnalisation dans le domaine du verbe », CMLF 2012, SHS Web of Conferences 1, p. 43-80, DOI: http://dx.doi.org/10.1051/shsconf/20120100341.

KURYLOWICZ J., 1965, « The evolution of grammatical categories », in Esquisses linguistiques (1975), Fink : Münich, p. 38-54.

LeHMANN C., 1985, « Grammaticalization : Synchronic Variation and Diachronic Change », Lingua E Stile, 20, p. 303-318.

MeILlet A., 1912, «L’évolution des formes grammaticales », Scientia, Rivista di scienza, 12(26). (réimprimé dans MeILlet A., 1958, Linguistique Historique et Linguistique générale, Paris: Champion, p. 130-149).

MARCHELLO-NIZIA C., 2006, Grammaticalisation et changement linguistique, Bruxelles, De Boeck.

Nicot J., 1606, Thresor de la langue françoise, tant ancienne que moderne, Douceur, Paris.

ŠTiCHAUER J., 2006, «La préposition dans, la locativité et la diachronie », Philologica Jassyensia, 2, p. 137-146.

ŠTICHAUER J., 2010, «Evolution des prépositions et emplois locatifs en francais préclassique et classique et la notion de locativité forte/faible », in ILIESCU M., SiLLER-RUnGGALDIER H. et DANLER P., $X X V^{e}$ CILPR Congrès International de Linguistique et de Philologie Romanes (Innsbruck, 3-8 septembre 2007), Berlin / New York : De Gruyter, t. 2, p. 565-574.

TRAugotT E.C., 2003, « Constructions in Grammaticalization », in JosEPH B.D. et JANDA R.D. (eds), The Handbook of Historical Linguistics, Oxford : Blackwell, p. 624-647.

VANDELOISE C., 1986, L'espace en français, Paris : Seuil.

ZIPF G., 1949, Human Behavior and the Principle of Least Effort, Cambridge, Addison-Wesley. 
Corpus utilisés :

- Le Monde

- Valibel (http://www.uclouvain.be/valibel.html)

- Frantext (http://www.atilf.fr/)

- $\quad$ DMF (http://www.atilf.fr/) 